\title{
Abnormal Repolarization in the Acute Myocardial Infarction Patients: A Frequency-Based Characterization
}

\author{
Corrado Giuliani, Angela Agostinelli, Sandro Fioretti, Francesco D. Nardo and Laura Burattini*
}

Department of Information Engineering, Università Politecnica delle Marche, Ancona, Italy

\begin{abstract}
Despite ST elevation having poor sensitivity for acute myocardial infarction (AMI), it remains the main electrocardiographic (ECG) repolarization index for AMI diagnosis. Aim of the present study was to propose a new $f 99$ index, defined as the frequency at which the repolarization normalized cumulative energy reaches $99 \%$, for ECG AMI discrimination from health with good sensitivity and good specificity. Evaluation of such $f 99$ index was performed on 12-standard-lead (I, II, III, aV1, aVr, aVf, V1 to V6) ECG recordings of 47 healthy controls and 108 acute myocardial infarction (AMI) patients. Repolarization dispersion caused $f 99$ distributions to be significantly lead dependent. In most leads (leads I, II, aV1, aVr, V2-V6), $f 99$ median value was lower in the healthy controls $(10-17 \mathrm{~Hz})$ than in the AMI patients $(12-38 \mathrm{~Hz})$ indicating higher frequency components (i.e. a more fragmented repolarization) in the latter population. AMI patients from healthy controls discrimination by $f 99$, evaluated in terms of sensitivity (Se) and specificity (Sp), was also lead dependent. Single-lead analysis indicated leads I $(\mathrm{Se}=80 \%, \mathrm{Sp}=77 \%)$ and aV1 $(\mathrm{Se}=84 \%, \mathrm{Sp}=74 \%)$ as optimal. Instead, lead-system analysis, performed to overcome dispersion issues, provided the best results when averaging over the 6 precordial leads $(\mathrm{Se}=81 \%$ and $\mathrm{Sp}=74 \%$ ). In conclusion, our new $f 99$ index appears as a promising tool for noninvasively and reliably discriminate AMI patients from healthy subjects.
\end{abstract}

Keywords: Digital electrocardiography, ECG repolarization index, myocardial infarction, repolarization variability, T-wave frequency content.

\section{INTRODUCTION}

Defects in the cardiac repolarization are known to be associated to several life-threatening diseases [1-4]. In the electrocardiogram (ECG) such defects appear as abnormalities of the ST segment and T-wave, which can be non-invasively characterized by means of indexes. The most popular ECG repolarization indexes are the QT interval $[2,3]$, the ST elevation $[5,6]$ and the T-wave alternans $[4,7]$. Additional morphological indexes present in the literature include the T-wave duration parameters [8,9], the T-wave amplitude parameters [10,11] and others [12-16]. Abnormalities in the ECG repolarization morphology are reflected, in the frequency domain, in a variation of the $\mathrm{T}$-wave frequency content. Though, repolarization indexes based on this feature have only occasionally been proposed [17-19].

The acute myocardial infarction (AMI: a disease in which the blood stops flowing properly to part of the heart and the heart is injured because not receiving enough oxygen) is one of the leading causes of death and disability in the world [20]. Even though in most cases ST elevation is a result of non-AMI causes [5] and several other ECG repolarization abnormalities have been observed in the AMI patients $[2,18$, $19,21,22]$, the ST elevation remains the ECG repolarization

*Address correspondence to this author at the Department of Information Engineering, Polytechnic University of Marche, 60131 Ancona, Italy; Tel: (39) 071220 4461; Fax (39) 071220 4224;

E-mail: 1.burattini@univpm.it index mainly used to diagnose the presence of an AMI [23,24] since initial ST elevation is part of the classic evolutionary pattern of the AMI [25]. Still, ST elevation has poor sensitivity for AMI given that up to $50 \%$ of patients exhibit atypical repolarization changes which may include isolated ST depression, $\mathrm{T}$ inversion or even normal ECG [24]. In order to significantly improve the rate of correct AMI diagnosis from ECG tracings, new repolarization parameters are needed. Thus, aim of the present study was to propose a new repolarization index based on the T-wave frequency content, termed $f 99$, able to discriminate AMI patients from healthy subjects with both a good sensitivity and a good specificity. Being defined in the frequency domain, $f 99$ computation requires automatic analysis of the ECG signals. Often, many of the above-mentioned timedomain repolarization parameters are still manually evaluated. Compared to human evaluation, automatic ECG analysis allows a more objective characterization of the ECG features by eliminating the subject-related variations of the measures, even though some inter-method variations may persist [26,27].

\section{METHODS}

\subsection{Clinical Data}

Our clinical data consisted of short (30 s to $2 \mathrm{~min}$ ) 12-standard-lead (I, II, III, aV1, aVr, aVf, V1 to V6) digital ECG recordings (1 KHz sampling frequency) from 47 healthy controls and 108 myocardial infarction patients (AMI), all belonging to the PTB Diagnostic Physionet 
database (www.physionet.org). Among the AMI patients, 49 were affected by anterior acute myocardial infarction (ANAMI) and 59 by inferior acute myocardial infarction (INAMI). Additional clinical information relative to the two populations is reported in Table $\mathbf{1}$.

\subsection{Repolarization Characterization}

Preprocessing. Before T-wave frequency-content evaluation, ECG tracings underwent a preprocessing stage consisting of $F_{s}=200 \mathrm{~Hz}$ resampling, low-frequency $(\leq 0.5$ $\mathrm{Hz}$ ) noise removal and $50 \mathrm{~Hz}$ line noise removal. Eventually, after R-peak detection, a 20-beat 12-lead ECG window characterized by stable HR (RR-interval standard deviation $<10 \%$ of mean RR) and no ectopic beats and artifacts was randomly extracted from each ECG tracing.

Repolarization frequency-content characterization. Repolarization frequency-content evaluation was performed in each ECG lead independently. From the median beat, computed using the 20 available beats, the repolarization onset (RepOn) and offset (RepOff) were identified as:

RepOn $=70 \mathrm{~ms}$ from the R - peak

RepOff $=0.3 \sqrt{\text { medianRR }} \cdot 1000 \mathrm{~ms}$ from the RepOn point.

Eq. (2) is an adjustment of an empirical formula [28] finalized to avoid cases of $\mathrm{P}$-wave inclusion in the $\mathrm{T}$-wave window, and median RR (s) is the median RR interval. The median repolarization waveform was then forced to be $260 \mathrm{~ms}$ long by opportune resampling. Eventually, the repolarization signal (RPS) was constructed by zero padding everything outside the resampled median repolarization waveform till 1 second.

$R P S$ frequency-content evaluation was performed by computing the Fourier power spectrum $\left(P S_{R P S}(k)\right.$; Eq. 3$)$ and energy signal $\left(E_{R P S}(k)\right.$; Eq. 4$)$ :

$$
\begin{aligned}
& P S_{R P S}(k)=\sum_{n=0}^{N_{S}-1} R P S(n) \cdot \exp -j 2 \pi \frac{k}{N_{s}} n \\
& E_{R P S}(k)=\sum_{m=0}^{k} P S_{R P S}(m)
\end{aligned}
$$

where $N_{s}$ is the number of samples $\left(N_{s}=200\right)$, and $\mathrm{n}$ and $\mathrm{k}$ are adimensional indexes to get time and frequency as $t_{n}=n \cdot\left(1 / F_{s}\right)=n \cdot 0.005 \mathrm{~s}$, with $n=1,2, \ldots N_{s}$, and $f_{k}=k \mathrm{~Hz}$, with $k=1,2, \ldots N_{s} / 2$, respectively. After having computed the total energy ( $E_{R P S}$ Total; Eq. 5), the $P S_{R P S}(k)$ and the $E_{R P S}(k)$ were normalized and expressed as percentages $\left(P S_{R P S} \%(k)\right.$ and $E_{R P S} \%(k)$, respectively; Eq. 6 and Eq. 7):

$$
\begin{aligned}
& E_{R P S-T o t a l}=\sum_{k=0}^{\frac{N_{s}}{2}-1} P S_{R P S}(k) \\
& P S_{R P S} \%(k)=\frac{P S_{R P S}(k)}{E_{R P S}-\text { Total }} \cdot 100 \\
& E_{R P S} \%(k)=\frac{E_{R P S}(k)}{E_{R P S}-\text { Total }} \cdot 100
\end{aligned}
$$

By definition, $E_{R P S} \%(k)$ is a monotonically increasing function which saturates at $100 \%$. The frequency at which $E_{R P S} \%$ first reaches or overcomes $99 \%$, called $f 99$, represents an index to characterize repolarization.

Abnormal repolarization identification. Abnormal repolarization was identified when $f 99$ exceeded a threshold defined as the $75^{\text {th }}$ percentile of the $f 99$ distribution over the healthy controls.

\subsection{Repolarization Analysis Types}

Two types of repolarization analysis were performed:

- Single-lead analysis. The $f 99$ values were computed in each ECG lead, and the abnormal repolarization identification criterion was then applied to their singlelead distributions.

- Lead-system analysis. The single-lead $f 99$ values were averaged over the 6 precordial and 12-standard-leads, and the abnormal repolarization identification criterion was then applied to the distributions of the averaged $f 99$ values.

Table 1. Clinical parameters. Mean \pm standard deviation values or number of occurrences are reported.

\begin{tabular}{|c|c|c|c|c|}
\hline & Healthy Controls (47) & ANAMI (49) & INAMI (59) & AMI (108) \\
\hline \hline Age (year) & $45 \pm 15$ & $60 \pm 11^{\circ}$ & $58 \pm 11^{\circ}$ & $59 \pm 11^{\circ}$ \\
\hline Gender (male) & $37(82 \%)$ & $39(80 \%)$ & $49(83 \%)$ & $32(30 \%)^{\circ}$ \\
\hline Hypertension & $0(0 \%)$ & $12(25 \%)^{\S}$ & $20(34 \%)^{\circ}$ & $9(8 \%)$ \\
\hline Obesity & $0(0 \%)$ & $4(8 \%)$ & $5(8 \%)^{*}$ & $12(20 \%)^{\S}$ \\
\hline Diabetes & $0(0 \%)$ & $4(8 \%)$ & $29(49 \%)^{\circ}$ & $16 \%)^{\S}$ \\
\hline Other pathologies & $0(0 \%)$ & $28(57 \%)^{\circ}$ & $81 \pm 17^{\S}$ & $57(53 \%)^{\circ}$ \\
\hline Heart rate (bpm) & $69 \pm 12$ & $82 \pm 15^{\circ}$ & & $81 \pm 16^{\circ}$ \\
\hline
\end{tabular}

* $\mathrm{P}<0.05$ when comparing against the healthy controls $\S \mathrm{P}<10^{-3}$ when comparing against the healthy controls ${ }^{\circ} \mathrm{P}<10^{-5}$ when comparing against the healthy controls 


\subsection{Algorithm Implementation and Simulation Test}

The $f 99$ algorithm was implemented in MATLAB and validated using simulated data with known input data. Specifically, a signal $y(t)$, constituted by the summation of two sinusoids $\mathrm{y}_{1}(\mathrm{t})$ and $\mathrm{y}_{2}(\mathrm{t})$, was considered:

$y(t)=y_{1}(t)+y_{2}(t)=A_{1} \cos \left(2 \pi f_{1} t\right)+A_{2} \cos \left(2 \pi f_{2} t\right)$.

Validation was performed by keeping constant the values of $f_{1}, f_{2}$, and $A_{1}\left(f_{l}=1 \mathrm{~Hz} ; f_{2}=15 \mathrm{~Hz} ; A_{l}=1\right.$ a.u. $)$, while the $A_{2}$ was varying from 0 to 1 a.u. (0.01 steps). Being $f 99$ the frequency at which the signal total energy $\left(E=E_{l}+E_{2}=A_{l}^{2}+\right.$ $A_{2}{ }^{2}$ ) reaches or overcomes $99 \%, f 99$ is correctly estimated if it equals $1 \mathrm{~Hz}$ till $A_{2}=A_{2}{ }^{*}$ and then becomes equal to $15 \mathrm{~Hz}$. $A_{2} *$ is obtained by putting $E_{l}=0.99 \cdot\left(E_{l}+E_{2}\right)$, which implies $0.99 \cdot\left(A_{2}^{*}\right)^{2}=(1-0.99) A_{1}^{2}$, that is:

$A_{2}^{*}=\sqrt{0.01 / 0.99}=0.1$

\subsection{Robustness Tests}

To evaluate $f 99$ robustness, three kinds of tests were considered:

- Robustness to dispersion. Single-lead $f 99$ distributions were compared to evaluate the presence of $f 99$ inter-lead dispersion.

- Robustness to repolarization window offset. The $f 99$ values were computed using the nominal RepOn and RepOff points (Eq. 1 and Eq. 2) and after a random $\pm 20 \mathrm{~ms}$ shift of RepOff from its nominal location. Results obtained in the two experiments were compared to investigate $f 99$ dependency on RepOff identification.

- Robustness to heart rate. The association between $f 99$ and corresponding HR was evaluated to investigate the dependency of the former parameter on the latter.

\subsection{Statistics}

Normality of parameters distributions was tested using the Lilliefors test. Parameters characterized by normal and not-normal distributions were compared using the T-test and the Wilcoxon rank-sum test, respectively. Binary parameters distributions were compared using the chi-square test or, when not possible (expected cell frequency <5), the Fisher exact one-tailed probability test. The Kruskal-Wallis test was used to perform the one-way ANOVA test to compare distributions over the leads. Lead-pairs comparison was performed using the multiple comparison procedure. Associations between two parameters distributions were evaluated using the correlation coefficient $(\rho)$. Eventually, $f 99$ ability to discriminate abnormal repolarization was evaluated using sensitivity $(S e)$, and specificity ( $S p$, fixed at $75 \%$ by the abnormal repolarization criterion defined above). Statistical significance level was 0.05 .

\section{RESULTS}

Simulation study. In our simulation study, $f 99$ resulted equal to $1 \mathrm{~Hz}$ for $A_{2}$ ranging from 0 to $A_{2}{ }^{*}=0.1$, and to $15 \mathrm{~Hz}$ for higher values of $A_{2}$ (Fig. 1), confirming the goodness of our 999 -algorithm MATLAB implementation.

Single-lead analysis. In most leads, the AMI patients' spectra were characterized by larger high-frequency components than the healthy controls' spectra (Fig. 2). Consequently, compared to the AMI patients, the healthy controls were usually characterized by $E_{R P S} \%$ curves saturating at lower frequencies (Fig. 3) and by significantly lower $f 99$ values (Table 2). Lead III was the only one showing an opposite trend, whereas aVf and V1 $f 99$ distributions did not differ significantly between the two populations (Table 2). Analogous findings were obtained when comparing ANAMI and INAMI subgroups against the healthy controls (Table 2). Ability of $f 99$ in discriminating

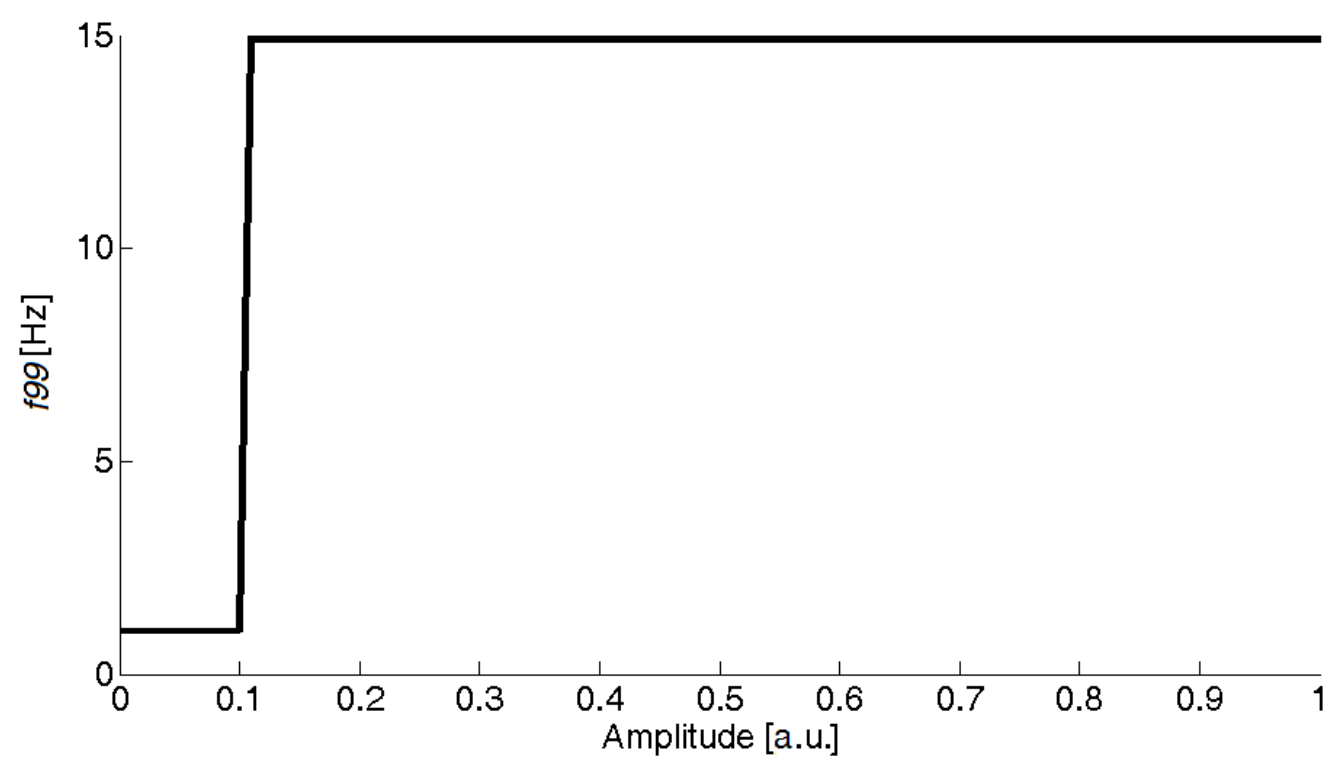

Fig. (1). Trend of $f 99$ computed from a simulated signal $y(t)$ constituted by the summation of two sinusoids $y_{1}(t)$ and $y_{2}(t)$ characterized by constant frequency values $\left(f_{l}=1 \mathrm{~Hz}, f_{2}=15 \mathrm{~Hz}\right.$, respectively), constant $y_{1}(t)$ amplitude $\left(A_{l}=1\right.$ a.u. $)$ and varying $y_{2}(t)$ amplitude $\left(A_{2}=0-1\right.$ a.u. $)$. 

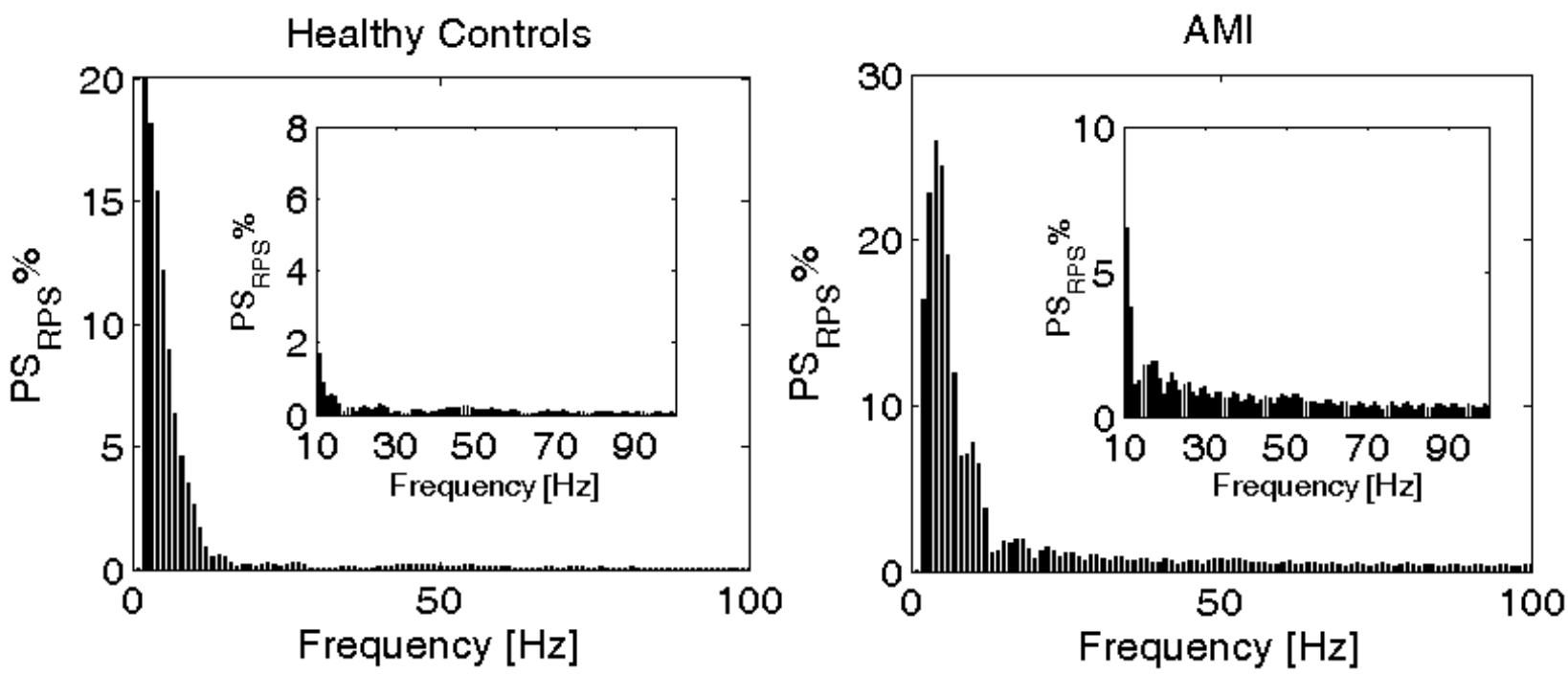

Fig. (2). Typical normalized spectra of a healthy control (panel a) and an AMI patient (panel b). Plots refer to lead V2. The inset represents a zoomed portion (from 10 to $100 \mathrm{~Hz}$ ) of the larger graph finalized to highlight high-frequency components that otherwise would remain hidden because of the presence of much higher-amplitude low-frequency (below $10 \mathrm{~Hz}$ ) components.

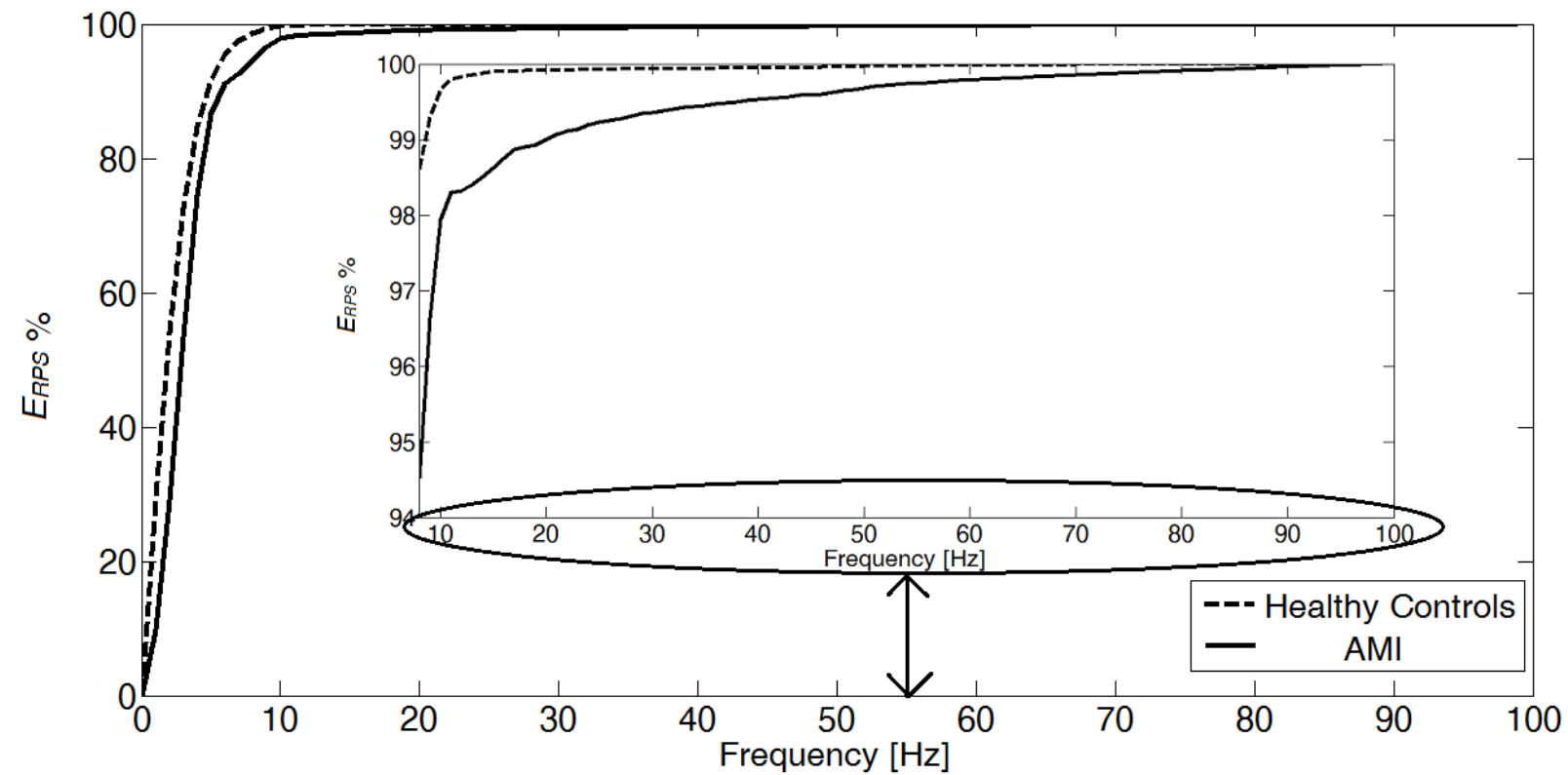

Fig. (3). Typical normalized energy curves of a healthy control (dotted line) and an AMI patient (solid line). Plots refer to lead V2 (same subjects of Fig. 2). The inset represents a zoomed portion (from 10 to $100 \mathrm{~Hz}$ ) of the larger graph finalized to highlight the distance between the energy graphs of the healthy controls and AMI patients.

pathological from healthy conditions, evaluated in terms of Se and Sp, was lead dependent (Table 3). Optimal leads could also vary with AMI subgroup. Leads I and aVl discriminated both the ANAMI and INAMI patients from the healthy controls. Instead, lead V2 discriminated the ANAMI group better than the INAMI group, while lead V5 vice versa (Table 3).

Lead-system analysis. Mean $f 99$ values, averaged over the 6 precordial and 12-standard-leads, were significantly lower in the healthy controls than the AMI patients (Table 2). However, discrimination between the two populations was superior when averaging over the 6 precordial leads than over the 12-standard-leads (Table 3). Analogous results were obtained when analyzing the two AMI subgroups independently (Tables $\mathbf{2}$ and $\mathbf{3}$ ).

Robustness to dispersion. No specific lead showed significantly higher or lower $f 99$ values compared to all other leads (Table 2). However, statistically significant differences were identified among couples of leads, so that final values of $S e$ and $S p$ were indeed significantly lead dependent (Table 3).

Robustness to repolarization window offset. Distributions of $f 99$ parameter were very robust to changes in the RepOff identification in all populations (Table 4). Consequently, values of $S e$ and $S p$ were very close to those obtained using the RepOff nominal value (Table 5). 
Table 2. Values of $50^{\text {th }}\left[25^{\text {th }}, 75^{\text {th }}\right]$ percentiles of the $f 99$ distributions.

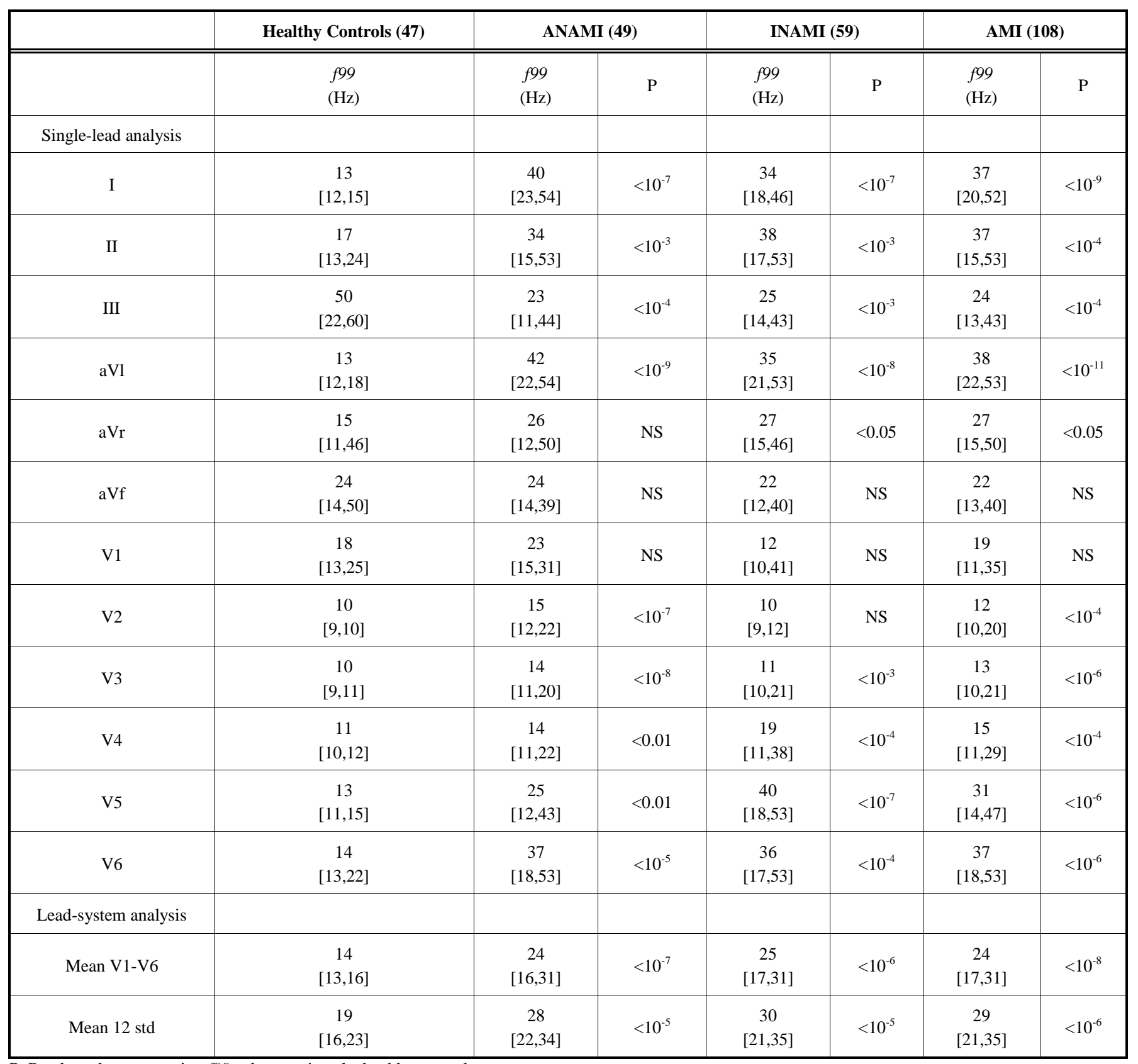

P: P-value when comparing $f 99$ values against the healthy controls NS: not statistically significant $(\mathrm{P} \geq 0.05)$

Table 3. Values of $f 99$ threshold over which abnormal repolarization is recognized together with corresponding values of sensitivity (Se) and specificity (Sp).

\begin{tabular}{|c|c|c|c|c|c|}
\hline & & Healthy Controls & ANAMI & INAMI & Se \\
\hline & $f 99$ threshold (Hz) & Sp & Se & \\
\hline \hline Single-lead analysis & & & & $78 \%$ \\
\hline I & 15.0 & $77 \%$ & $61 \%$ & $61 \%$ \\
\hline II & 24.3 & $74 \%$ & $60 \%$ & $61 \%$ \\
\hline III & 60.0 & $77 \%$ & $6 \%$ & $7 \%$ \\
\hline
\end{tabular}


Table 3. Contd.....

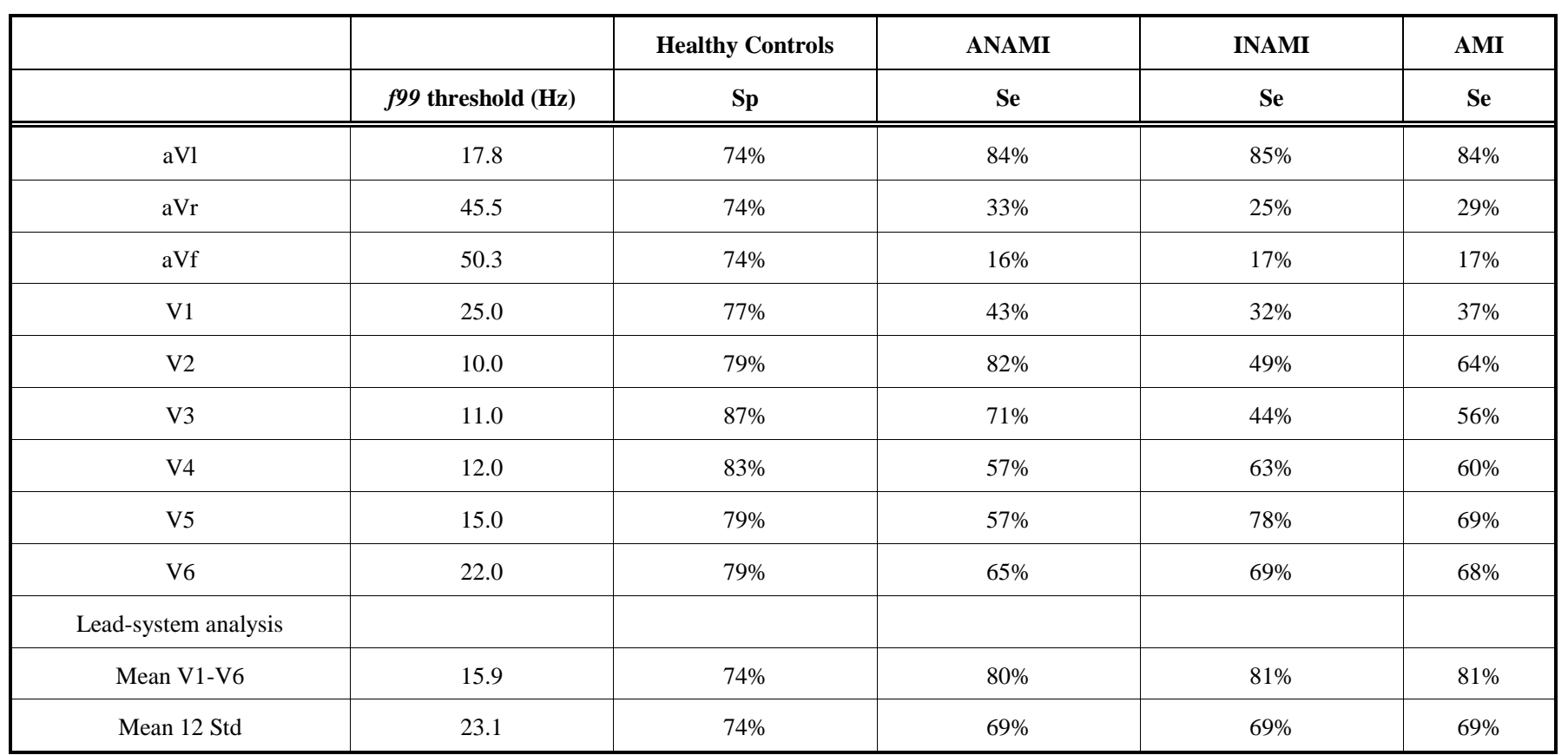

Table 4. Values of the correlation coefficient ( $\rho$ ) between $f 99$ distributions obtained using nominal and randomly shifted (within $\pm 20 \mathrm{~ms})$ RepOff points.

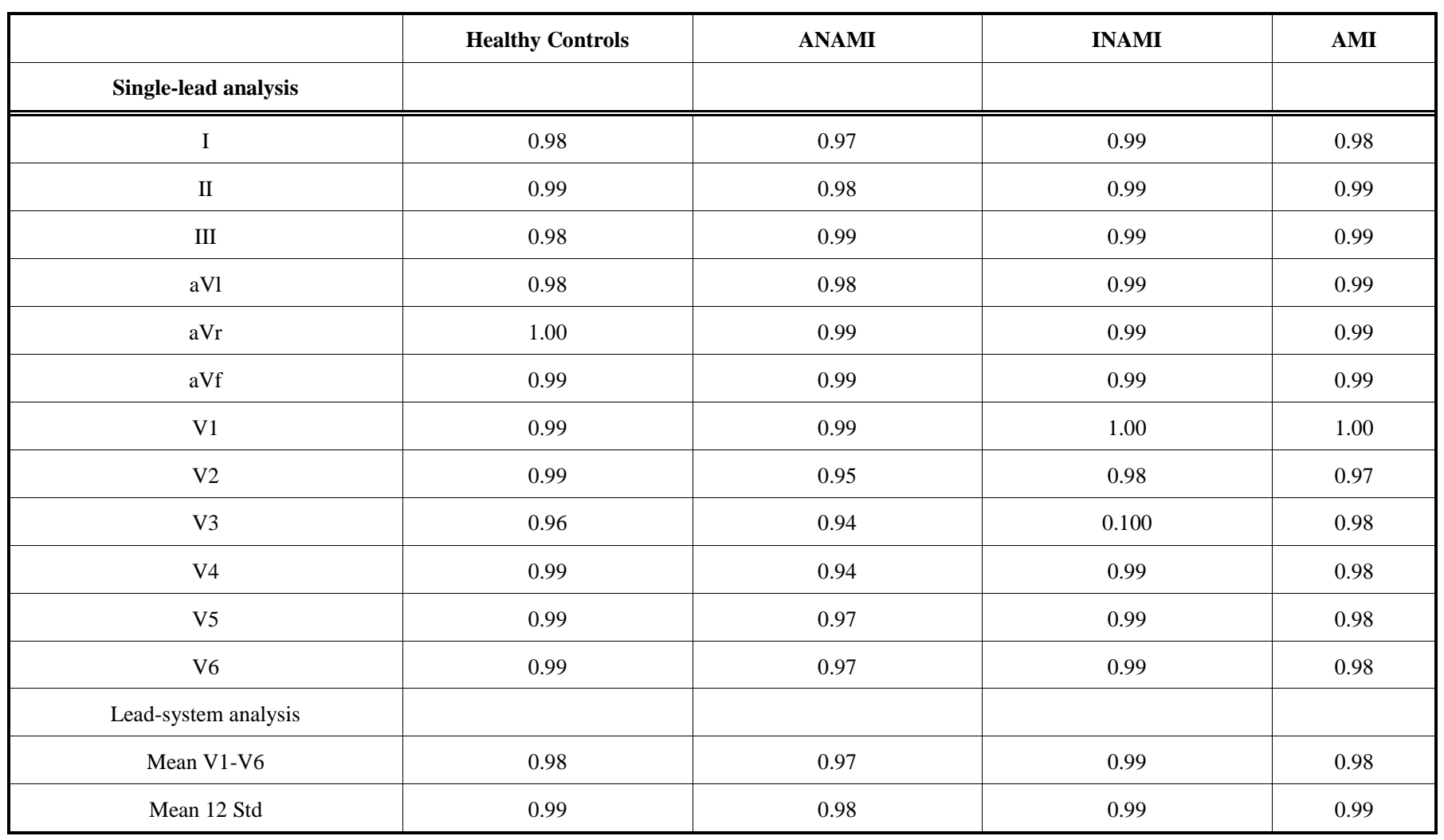

Robustness to heart rate. In all populations, $f 99$ values were substantially independent from HR (Table 6).

\section{DISCUSSION}

This study proposes $f 99$ as an innovative frequency-based index for repolarization characterization and AMI patients 
Table 5. Values of $f 99$ threshold over which abnormal repolarization is recognized together with corresponding values of sensitivity (Se) and specificity (Sp) when RepOff was randomly shifted within $\pm 20 \mathrm{~ms}$ from its nominal value.

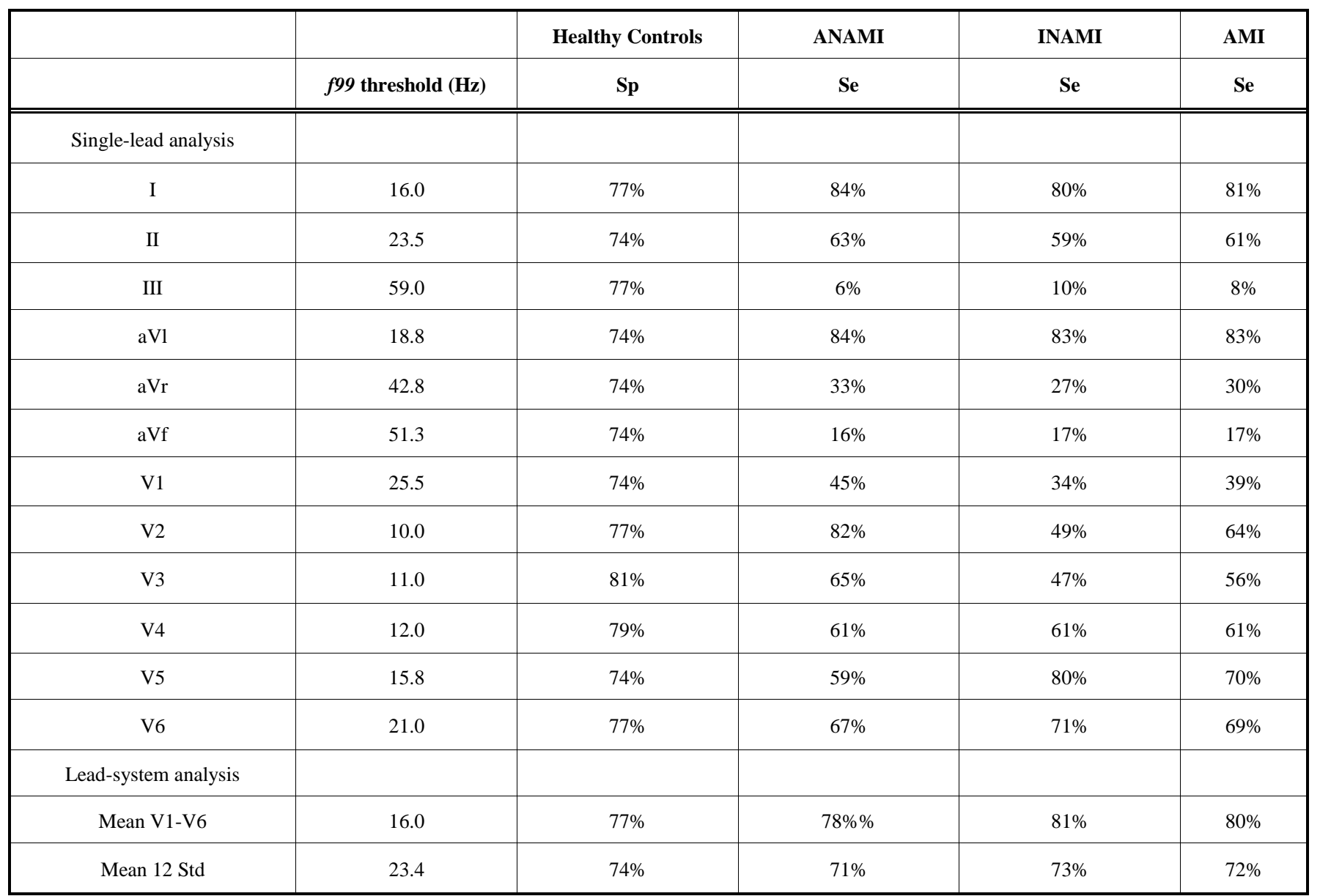

discrimination from healthy subjects. The algorithm for $f 99$ computation was tested on simulated data to confirm its proper functioning. Instead, $f 99$ clinical usefulness was evaluated on ECG tracings from 47 healthy controls and 108 AMI patients (49 ANAMI e 59 INAMI), who are known to show abnormal repolarization [17, 21, 22].

The $f 99$ index, defined as the frequency at which the repolarization normalized cumulative energy reaches $99 \%$, was computed in each one of the 12-standard-leads independently, and averaged over the 6 precordial and 12-standard-leads. In most leads (9 out of 12, Table 2), f99 median values was significantly lower for the healthy controls than for the AMI patients. This finding implies that AMI T-waves have a normalized power spectrum characterized by higher frequency components which indicate a more fragmented repolarization. On this basis, $f 99$ was used to discriminate abnormal from normal repolarization. More specifically, abnormal repolarization was identified when $f 99$ exceeded a threshold defined as the $75^{\text {th }}$ percentile of the $f 99$ distribution over the healthy controls. Such threshold definition implies a nominal $S p$ of $75 \%$. However, actual values of $S p$ were either slightly lower $(74 \%$, Table 3) or higher $(77 \%-87 \%$, Table 3). Lower values of $S p$ are due to the fact that the healthy-control population counts less than 100 subjects. Instead, higher values of $\mathrm{S} p$ are due to the fact that there can be more than one healthy control characterized by the same $f 99$ value immediately under threshold. $S e$ values were strongly lead dependent (Se=6\%-85\%, Table 3), indicating a significant repolarization dispersion identified by $f 99$. Leads I and aVl were able to significantly discriminate abnormal from normal repolarization, independently of the infarct location (lead I: $S e=78 \%-82 \%, S p=77 \%$; lead aVl: $S e=84 \%-85 \%$, $S p=74 \%$, Table 3). Instead, lead V2 allowed identification of abnormal repolarization only in the ANAMI patients $(S e=82 \%, S p=79 \%$, Table 3) while lead V5 only in the INAMI patients $(S e=78 \%, S p=79 \%$, Table 3). These findings indicate that $f 99$ is characterized by a certain amount of dispersion, similarly to what happens for other repolarization indexes $[29,30]$. Since the leads provide spatial information about the heart's electrical activity in different directions, the optimal lead for discriminating abnormal repolarization by f99 may depend on the infarct location. More specifically, leads closer to the AMI location will identify the disease better than the others.

Since it is not possible to know a priori which will be the optimum lead to identify an AMI, to overcome issues related to repolarization dispersion, values of $f 99$ averaged over the 
Table 6. Values of the correlation coefficient ( $\rho)$ between $f 99$ distributions and heart rate.

\begin{tabular}{|c|c|c|c|c|}
\hline & Healthy Controls & ANAMI & INAMI & AMI \\
\hline \multicolumn{5}{|c|}{ Single-lead analysis } \\
\hline II & $0.42 *$ & $0.41 *$ & 0.11 & $0.22 *$ \\
\hline aVl & $0.46^{*}$ & $0.37 *$ & 0.16 & $0.24 *$ \\
\hline $\mathrm{aVr}$ & 0.14 & $0.36^{*}$ & 0.04 & 0.18 \\
\hline $\mathrm{aVf}$ & $0.31^{*}$ & $0.52 *$ & 0.09 & $0.26^{*}$ \\
\hline V1 & 0.03 & 0.14 & -0.05 & 0.01 \\
\hline V4 & 0.11 & 0.19 & 0.25 & $0.21 *$ \\
\hline V5 & 0.20 & $0.35^{*}$ & 0.07 & 0.16 \\
\hline V6 & $0.34 *$ & 0.18 & -0.06 & 0.04 \\
\hline \multicolumn{5}{|c|}{ Lead-system analysis } \\
\hline Mean V1-V6 & 0.20 & $0.33^{*}$ & 0.05 & 0.14 \\
\hline Mean 12 Std & $0.36^{*}$ & $0.56^{*}$ & 0.12 & $0.29 *$ \\
\hline
\end{tabular}

*Statistically significant $(\mathrm{P}<0.05)$

6 precordial and 12-standard-leads were provided. Between these two averaged indexes, the former discriminated abnormal repolarization $(S e=80 \%-81 \%, S p=74 \%$, Table 3) better than the latter $(S e=69 \%, S p=74 \%$, Table 3) since the f99 distributions were showing a more homogeneous trend among the precordial leads than the limb and augmentedlimb leads (Table 2). Thus, lead-system analysis showed that the 6 precordial leads were superior to the 12-standard-leads in discriminating abnormal repolarization, even though the single-lead analysis showed that the optimal lead could not be precordial.

The $f 99$ index, either measured on a single lead or averaged over a lead system, is statistically independent from the exact determination of the repolarization window (Tables $\mathbf{4}$ and 5), and does not require the exact identification of the T-wave offset, usually very difficult in clinical cases [9]. T-wave offset estimation can indeed occur with a few tens of $\mathrm{ms}$ of tolerance (Table 5). In addition, $f 99$ was independent from HR (Table 6). In general, repolarization significantly depends on HR [10] so that some indexes, like the popular QT, may require a correction to adjust to it. The f99 independency of HR is due to the fact that such adjustment is performed during the construction of the RPS (see Methods), which requires a stretch or a compression of the repolarization segment to match a length of $260 \mathrm{~ms}$ (assumed as standard), and a subsequent zero padding to reach a $1 \mathrm{~s}$ length for each heart cycles. Thus, the normalized power spectrum does not provide the frequency content of the real $\mathrm{T}$-wave, but rather the amplitude of the harmonics after forcing the fundamental frequency to be at $1 \mathrm{~Hz}$. In other words, $f 99$ although expressed in $\mathrm{Hz}$ for simplicity and clarity, represents the number of harmonics the normalized cumulative energy needs to reach $99 \%$.

\section{CONCLUSION}

The present study proposed a new $f 99$ index, defined as the frequency at which the repolarization normalized cumulative energy reaches 99\%, for ECG AMI discrimination from health. Evaluation of such $f 99$ index was performed on 12-standard-lead ECG recordings of 47 healthy controls and 108 AMI patients. Repolarization dispersion caused $f 99$ distributions to be significantly lead dependent indicating that optimal lead for discriminating abnormal repolarization may depend on the infarct location. To overcome dispersion issues, lead-system analysis was performed by averaging $f 99$ over the 6 precordial leads and proved to be able to identify the presence of AMI with good sensitivity and specificity. Thus, our new $f 99$ index appears as a promising tool for non-invasively and reliably discriminate AMI patients from healthy subjects. 


\section{LIST OF ABBREVIATIONS}

\begin{tabular}{|c|c|c|}
\hline AMI & $=$ & acute myocardial infarction \\
\hline ANAMI & $=$ & anterior acute myocardial infarction \\
\hline$E_{R P S}(k)$ & $=$ & RPS energy \\
\hline$E_{R P S \_}$Total & $=$ & $R P S$ total energy \\
\hline$E_{R P S} \%(k)$ & $=$ & $\begin{array}{l}\text { RPS normalized and expressed as } \\
\text { percentage energy }\end{array}$ \\
\hline$f 99$ & $=$ & $\begin{array}{l}\text { frequency at which } \mathrm{E}_{R P S} \% \text { reaches } \\
\text { or overcomes } 99 \%\end{array}$ \\
\hline HR & $=$ & heart rate \\
\hline INAMI & $=$ & inferior acute myocardial infarction \\
\hline mean V1-V6 & $=$ & $\begin{array}{l}\text { mean } f 99 \text { values averaged over the } 6 \\
\text { precordial leads }\end{array}$ \\
\hline mean 12 std & $=$ & $\begin{array}{l}\text { mean } f 99 \text { values averaged over the } \\
12 \text { standard leads }\end{array}$ \\
\hline$P S_{R P S}(k)$ & $=$ & $R P S$ power spectrum \\
\hline$P S_{R P S} \%(k)$ & $=$ & $\begin{array}{l}\text { RPS normalized and expressed as } \\
\text { percentage power spectrum }\end{array}$ \\
\hline $\mathrm{RepOn}$ & $=$ & repolarization onset \\
\hline RepOff & $=$ & repolarization offset \\
\hline RPS & $=$ & repolarization signal \\
\hline SCD & $=$ & sudden cardiac death \\
\hline
\end{tabular}

\section{ETHICAL APPROVAL}

All Physionet databases have been fully deidentified (anonymized) and may be used without further institutional review board approval.

\section{CONFLICT OF INTEREST}

C. Giuliani, A. Agostinelli and F. Di Nardo have no financial and/or personal relationships with people or organizations that could inappropriately influence (bias) this work.

L. Burattini and S. Fioretti declare their partnership to the academic spin-off B.M.E.D. SRL (Bio-Medical Engineering Development, Department of Information Engineering, Polytechnic University of Marche, Ancona, Italy, www.bmed-bioengineering.com).

\section{ACKNOWLEDGEMENTS}

Declared none.

\section{REFERENCES}

[1] C. Antlzevitch, "Role of spatial dispersion of repolarization in inherited and acquired sudden cardiac death syndromes", Am. J. Physiol. Heart Circ. Physiol., vol. 293, pp. 2024-2038, Oct. 2007.

[2] H. Lindekleiv, T. Wilsgaard, P.W. Macfarlane, and M.L. Løchen, "QT Interval and the risk of myocardial infarction and all-cause death: a cohort study", J. Cardiovasc. Electrophysiol., vol. 23, pp. 846-852, Aug. 2012.

[3] Y. Zhang, W.S. Post, E. Blasco-Colmenares, D. Dalai, G.F. Tomaselli, and E. Guallar, "Electrocardiographic QT interval and mortality: a meta-analysis", Epidemiology, vol. 22, pp. 660670, Sept. 2011.

[4] J. Leino, M. Minkkinen, T. Nieminenl, T. Lehtimäki, J. Viik, R. Lehtinen, K. Nikus, T. Kööbi, V. Turjanmaa, R.L. Verrier, and M. Kähönenm, "Combined assessment of heart rate recovery and T-wave alternans during routine exercise testing improves prediction of total and cardiovascular mortality: the Finnish Cardiovascular Study", Heart Rhythm, vol. 6, no. 12, pp. 765-1771, Dec. 2009.

[5] L.A. Otto, and T.P. Aufderheide, "Evaluation of ST segment elevation criteria for the prehospital electrocardiographic diagnosis of acute myocardial infarction", Ann. Emerg. Med., vol. 23, pp. 17 24, Jan. 1994.

[6] W.J. Brady, A.D. Perron, E.A. Ullman, S.A. Syverud, C. Hostege, R. Riviello, and C. Ghammaghami, "Electrocardiographic ST segment elevation: a comparison of AMI and non-AMI ECG syndromes", Am. J. Emerg. Med., vol. 20, pp. 609-612, Nov. 2002.

[7] S. Man, P.V. De Winter, A.C. Maan, J. Thijssen, C.J. Borleffs, W.P. van Meerwijk, M. Bootsma, L. van Erven, E.E. van der Wall, M.J. Schalij, L. Burattini, R. Burattini, and C.A. Swenne, "Predictive power of T-wave alternans and of ventricular gradient hysteresis for the occurrence of ventricular arrhythmias in primary prevention ICD patients", J. Electrocardiol., vol. 44, pp. 453-459, July-August 2011.

[8] J.S. Hevia, C. Antzelevitc, F. Tornés Bárzaga, M. Dorantes Sánchez, F. Dorticós Balea, R. Zayas Molina, M.A. Quiñones Pérez, and Y. Fayad Rodríguez, "Tpeak-Tend and Tpeak-Tend dispersion as risk factors for ventricular tachycardia/ventricular fibrillation in patients with the Brugada syndrome", J. Am. Coll. Cardiol., vol. 47, pp. 1828-1834, May 2006.

[9] J.P. Couderc, W. Zareba, A.J. Moss, N. Sarapa, J. Morganroth, and B. Darpo, "Identification of Sotalol-induced changes in repolarization with $\mathrm{T}$-wave area-based repolarization duration parameters", J. Electrocardiol., vol. 36, pp. 115-120, Dec. 2003.

[10] C. Antzelevitch, "Ionic molecular and cellular bases of QT-interval prolongation and torsade de pointes", Europace, vol. 9, pp. 4-15, Sept. 2007.

[11] J.P. Couderc, S. McNitt, J. Xia, W. Zareba, and A.J. Moss, "Repolarization morphology in adult LQT2 carriers with borderline prolonged QTc interval", Heart Rhythm, vol. 3, pp. 1460-1466, Dec. 2006

[12] L. Aro, O. Anttonen, J.T. Tikkanen, M.J. Junttila, T. Kerola, H.A. Rissanen, A. Reunanen, and H.V. Huikuri, "Prevalence and prognostic significance of $\mathrm{T}$-wave inversions in right precordial leads of a 12-lead electrocardiogram in the middle-aged subjects", Circulation, vol. 125, pp. 2572-2577, May 2012.

[13] M. Haïssaguerre, N. Derval, F. Sache, L. Jesel, I. Deisenhofer, L. de Roy, J.L. Pasquié, A. Nogami, D. Babuty, S. Yli-Mayry, C. De Chillou, P. Scanu, P. Mabo, S. Matsuo, V. Probst, S. Le Scouarnec, P. Defaye, J. Schlaepfer, T. Rostock, D. Lacroix, D. Lamaison, T. Lavergne, Y. Aizawa, A. Englund, F. Anselme, M. O'Neill, M. Hocini, K.T. Lim, S. Knecht, G.D. Veenhuyzen, P. Bordachar, M. Chauvin, P. Jais, G. Coureau, G. Chene, G.J. Klein, and J. Clémenty, "Sudden cardiac arrest associated with early repolarization", N. Engl. J. Med., vol. 358, pp. 2016-2023, May 2008.

[14] M. Zabel, B. Acar, T, Klingenheben, M. R. Franz, S. H. Hohnloser, and M. Malik, "Analysis of 12-lead T-wave morphology for risk stratification after myocardial infarction", Circulation, vol. 102, pp. 1252-1257, Sep. 2000.

[15] L. Burattini, and W. Zareba, "Time-domain analysis of beat-to-beat variability of repolarization morphology in patients with ischemic cardiomyopathy", J. Electrocardiol., vol. 32, pp. 166-171, 1999.

[16] J.P. Couderc, W. Zareba, L. Burattini, and A.J. Moss, "Beat-to-beat repolarization variability in LQTS patients with the SCN5A sodium channel gene mutation", Pacing Clin. Electrophysiol., vol. 22, pp. 1581-1592, Nov. 1999

[17] P. Steinbigler, R. Haberl, K. Nespithal, A. Spiegl, I. Schmücking, and G. Steinbeck, "T-wave spectral variance: A new method to determine inhomogeneous repolarization by $\mathrm{T}$-wave beat-to-beat 
variability in patients prone to ventricular arrhythmias", J. Electrocardiol., vol. 30, pp. 137-144, 1998.

[18] L. Burattini, and C. Giuliani, T-wave Frequency Content Evaluation in Healthy Subjects and Patients Affected by Myocardial Infarction, In: Signal Processing: New Research, G.R. Naik, Nova Science Publishers Inc., 2013, pp. 79-93.

[19] G. Bakul and U.S. Tiwary, "Automated risk identification of myocardial infarction using relative frequency band coefficient (RFBC) features from ECG", Open Biomed. Eng. J., vol. 4, pp. 217-222, 2010.

[20] V.L. Roger, A.S. Go, D.M. Lloyd-Jones, E.J. Benjamin, J.D. Berry, W.B. Borden, D.M. Bravata, S. Dai, E.S. Ford, C.S. Fox, H.J. Fullerton, C. Gillespie, S.M. Hailpern, J.A. Heit, V.J. Howard, B.M. Kissela, S.J. Kittner, D.T. Lackland, J.H. Lichtman, L.D. Lisabeth, D.M. Makuc, G.M. Marcus, A. Marelli, D.B. Matchar, C.S. Moy, D. Mozaffarian, M.E. Mussolino, G. Nichol, N.P. Paynter, E.Z. Soliman, P.D. Sorlie, N. Sotoodehnia, T.N. Turan, S.S. Virani, N.D. Wong, D. Woo, and M.B. Turner, "Executive summary: heart disease and stroke statistics--2012 update: a report from the American Heart Association", Circulation, vol. 125, pp. 188-197, Jan. 2012.

[21] L. Burattini, S. Bini, and R. Burattini, "Repolarization alternans heterogeneity in healthy subjects and acute myocardial infarction patients", Med. Eng. Phys., vol. 34, pp. 305-312, April 2012.

[22] Y. Aizawa, M. Jastrzebski, T. Ozawa, K. Kawecka-Jaszcz, P. Kukla, W. Mitsuma, M. Chinushi, T. Ida, Y. Aizawa, K. Ojima, M. Tagawa, S. Fujita, M. Okabe, K. Tsuchida, Y. Miyakita, H. Shimizu, S. Ito, T. Imaizumi, and K. Toba, "Characteristics of electrocardiographic repolarization in acute myocardial infarction complicated by ventricular fibrillation", J. Electrocardiol, vol. 45, pp. 252-259, May 2012.

[23] P.T. O'Gara, F.G. Kushner, D.D. Ascheim, D.E. Jr. Casey, M.K. Chung, J.A. de Lemos, S.M. Ettinger, J.C. Fang, F.M. Fesmire, B.A. Franklin, C.B. Granger, H.M. Krumholz,
J.A. Linderbaum, D.A. Morrow, L.K. Newby, J.P. Ornato, N. Ou, M.J. Radford, J.E. Tamis-Holland, C.L. Tommaso, C.M. Tracy, Y.J. Woo, D.X. Zhao, J.L. Anderson, A.K. Jacobs, J.L. Halperin, N.M. Albert, R.G. Brindis, M.A. Creager, D. DeMets, R.A. Guyton, J.S. Hochman, R.J. Kovacs, F.G. Kushner, E.M. Ohman, W.G. Stevenson, and C.W. Yancy, "2013 ACCF/ AHA guideline for the management of ST-elevation myocardial infarction: a report of the American College of Cardiology Foundation/American Heart Association Task Force on Practice Guidelines.", Circulation vol. 127, pp. 362-425, Jan. 2012.

[24] B. Menown, G. Mackenzie, and A.A. Adgey, "Optimizing the initial 12-lead electrocardiographic diagnosis of acute myocardial infarction", Eur. Heart J., vol. 21, pp. 275-283, Feb. 2000.

[25] H.E.B. Pardee, "An electrocardiographic sign of coronary artery obstruction", Arch. Intern. Med., vol. 26, pp. 244-257, Aug. 1920.

[26] M. Malik, "Errors and misconceptions in ECG measurement used for the detection of drug induced QT interval prolongation", $J$. Electrocardiol., vol. 37, pp. 25-33, 2004.

[27] S.W. Smith, "ST segment elevation differs depending on the method of measurement", Acad. Emerg. Med., vol. 13, pp. 406412, April 2006.

[28] L. Burattini, W. Zareba, and A.J. Moss, "Correlation method for detection of transient T-wave alternans in digital Holter ECG recordings", Ann. Noninvasive Electrocardiol, vol. 4, pp. 416-424, Oct. 1999.

[29] C. Cowan, K. Yusoff, M. Moore, P.A. Amos, A.E. Gold, J.P. Bourke, S. Tansuphaswadikul, and R.W. Campbell, "Importance of lead selection in QT interval measurement", Am. J. Cardiol., vol. 61, pp. 83-87, Jan. 1988.

[30] Burattini, S. Man, R. Burattini, and C.A. Swenne, "Comparison of standard vs. orthogonal ECG leads for T-wave alternans identification", Ann. Noninvasive Electrocardiol., vol. 17, pp. 130140, April 2012.

This is an open access article licensed under the terms of the Creative Commons Attribution Non-Commercial License (http://creativecommons.org/licenses/by-nc/3.0/) which permits unrestricted, non-commercial use, distribution and reproduction in any medium, provided the work is properly cited. 